\title{
Factores de riesgo familiares asociados a la conducta suicida en adolescentes con trastorno depresivo
}

\author{
Paula Pavez ${ }^{a}$, N atalia Santandera, Jorge Carranza ${ }^{a}$, Pablo \\ Vera-Villarroel.
}

\section{Familial risk factors for suicide among adolescents with depression}

Background: Suicide is the second or third cause of death among Chilean teenagers aged 15 to 19 years. Aim: To evaluate familial risk factors associated to suicidal attempt in adolescents with a depressive disorder. Material and methods: Thirty two teenagers with depression, aged between 13 and 18 years, receiving psychological treatment in six public Mental Health Centers in Santiago and 32 fathers, mothers or tutors were evaluated. Beck's Depression Inventory and the Family Adaptability and Cohesion Evaluation Scale (FACES III, family version) were applied to adolescents. Adults were evaluated by the Goldberg's General Health Questionnaire and the FACES III parents version. Results: No meaningful statistical association between adolescents suicidal attempt and family risk factors (family order, presence of family stressors and parent's mental health), was observed. However, there was a significant association between suicidal ideation of teenagers, their familiar adaptability and suicidal ideation of close relatives. Conclusions: Suicidal attempt in this group of teenagers was associated with familial adaptability and suicidal ideation by close relatives. Therefore close relatives must be integrated to the prevention programs for adolescents' suicide (Rev Méd Chile 2009; 137: 226-33).

(Key words: Adolescent; Depression; Suicide)

Recibido el 25 de enero, 2008. Aceptado el 3 de noviembre, 2008.

Escuela de Psicología, Universidad de Santiago de Chile USACH

apsicólogos/as Universidad de Santiago de Chile.

$\overline{\text { Correspondencia a: Dr. Pablo Vera-Villarroel, Escuela de }}$ Psicología. Universidad de Santiago de Chile, USACH. Avenida Ecuador 3650, 3ํ Piso. Santiago. Fax: 56-2-7761986.

E mail: pablo.vera@usach.cl 
$\mathrm{E}$ n la actualidad, el suicidio es una problemática relevante a nivel internacional, debido a su alta prevalencia y a su progresivo aumento a través de las décadas ${ }^{1-3}$. Esta temática se torna aún más grave en la población adolescente, puesto que los suicidios en este grupo etáreo, son cada vez más frecuentes ${ }^{3}$, constituyéndose en la segunda o tercera causa de muerte en jóvenes de 15 a 19 años en Chile ${ }^{4-6}$.

Uno de los factores de riesgo más importante para la consecución de un suicidio es el intento suicida $3,7,8$. Se estima que entre $2 \%$ y $12 \%$ de la población joven ha tratado quitarse la vida ${ }^{3}$, constituyéndose en un motivo frecuente de consulta en salud mental ${ }^{10,11}$ y en un grave problema para la salud pública?

La investigación sobre los factores de riesgo para el intento suicida adolescente ha establecido una relación con desórdenes psiquiátricos ${ }^{1,3,9,12}$, como con la adversidad familiar ${ }^{3}$

Dentro de los factores psicológicos y psiquiátricos, la depresión ha jugado un papel preponderante en el intento suicida, donde más de 50\% de las muertes por suicidio se deben a este trastorno ${ }^{13}$. En los adolescentes con depresión existe un riesgo mayor de presentar intentos 7,11 .

En cuanto al ámbito familiar, se ha estudiado la relación de su funcionamiento con el intento suicida $^{4,14}$, destacándose los siguientes factores de riesgo: dificultades en las relaciones ${ }^{5}$; comunicación conflictiva ${ }^{3,5,15}$; bajos niveles de cercanía afectiva; altos niveles de control parental ${ }^{16,17}$; estructura familiar inestable; historia familiar con depresión ${ }^{3,18}$; desórdenes psiquiátricos $3,7,19,20$; abuso de sustancias $^{3}$; conducta suicida $3,11,20,21$ y conflictos legales del padre ${ }^{3}$, entre otros.

La relevancia de estudiar los factores de riesgo psicosociales asociados al intento suicida, integrando la variable individual (trastorno depresivo) como ambiental (adversidad familiar), se sustenta en el hecho que el intento suicida es un fenómeno multicausal, no atribuible a un suceso aislado o específico $^{22}$.

Consecuentemente, el objetivo de este estudio fue identificar y describir los factores de riesgo familiares que se relacionan con el intento suicida en adolescentes diagnosticados con trastorno depresivo. Para esto se estudió tanto los adolescentes, como a sus padres o tutores, con el fin de relacionar las variables anteriormente descritas.

\section{PARTICIPANTES Y MÉTODO}

La muestra fue de tipo no probabilística intencional. Fue extraída de seis centros públicos de salud mental de la ciudad de Santiago.

Los sujetos estudiados fueron 64 personas, de las cuales, 32 correspondieron a padre, madre o tutor; y 32 a adolescentes entre 13 y 18 años (con una media de 15,2 años y una desviación estándar de 1,6), diagnosticados con depresión mayor. Para seleccionar a los sujetos se consideró en primera instancia los diagnósticos previos realizados por los profesionales de los centros de salud mental. Posteriormente este diagnóstico, fue confirmado según los criterios del DSM-IV para trastorno depresivo mayor por los investigadores, incluyendo además la revisión de las fichas clínicas y el reporte del profesional tratante. Adicionalmente, los investigadores examinaron directamente a los pacientes de modo de corroborar el diagnóstico y las variables de inclusión. Posteriormente a los participantes se les aplicó el inventario de depresión de Beck que reafirmó el diagnóstico. De la misma forma, se descartó la presencia de sintomatología psicótica, trastorno orgánico, de conducta y relacionados con sustancias.

La totalidad de los jóvenes estudiados se encontraban entre un mes y un año y medio de tratamiento psicoterapéutico. De este grupo, 15 adolescentes (14 mujeres y 1 hombre) intentaron suicidarse con una media de 4,7 meses (tiempo promedio ocurrido desde el momento del intento suicida hasta el momento de la evaluación). La mayoría recibió atención médica psiquiátrica. El grupo que no intentó el suicidio correspondió a 10 mujeres y 7 hombres.

Los datos demográficos de la investigación como sexo, edad, escolaridad, se consignaron en una Ficha de Selección y Caracterización de la Muestra, la cual se describe en el apartado de instrumentos.

El nivel de rechazo de la muestra fue 24 sujetos entre adultos y jóvenes, que aunque cumpliendo los criterios de inclusión no pudieron participar por la gravedad de la situación familiar en ese momento o la inestabilidad emocional del adolescente.

Procedimiento. Para la recolección de datos se tomó contacto con los centros de salud mental, 
donde se solicitó información a los profesionales sobre pacientes que cumplieran con los requerimientos.

Los sujetos se seleccionaron a partir de la revisión de las fichas clínicas y se consultó a los profesionales a cargo de los jóvenes, para completar la ficha de caracterización de la muestra.

Posteriormente, se invitó a los sujetos con su padre, madre o tutor para la aplicación de los cuestionarios, que se realizó en forma individual y en una sola sesión (previamente firmaron un consentimiento informado).

La aplicación de los instrumentos fue realizada por parte de los investigadores, quienes poseían un entrenamiento previo en aplicación de escalas, así como en su interpretación y corrección.

Instrumentos. Se consideró como variables a medir en los adolescentes: depresión, funcionamiento familiar, percepción de estresores familiares, así como las variables intento suicida e ideación suicida. Mientras que en los padres se evaluó las variables de funcionamiento familiar, percepción de estresores familiares y la salud mental.

Para la evaluación de las variables se utilizaron los siguientes instrumentos:

a) El Inventario de Depresión de Beck $^{23}$ : esta escala evalúa la gravedad e intensidad de la sintomatología con una muy buena consistencia interna $(\alpha=0,83)^{24}$, similar a la obtenida en este estudio $(\alpha=0,80)$. Además se utilizó el ítem 9 del inventario para evaluar el nivel de ideación suicida.

b) FACES III; Family Adaptability and Cohesion Evaluation Escale ${ }^{25}$ : para evaluar el funcionamiento familiar, este instrumento entrega información sobre la percepción que cada miembro tiene de su familia en cuanto a la adaptabilidad y cohesión. Estudios previos exponen que presenta un alpha de Cronbach de 0,6526,27. Este material se desarrolló para estimar el funcionamiento familiar de acuerdo al Modelo Circunplejo de Olson, Russell y Sprenkle ${ }^{27,28}$, en donde el funcionamiento se entiende como el resultado de las características interaccionales de los miembros de la familia en dos áreas de la vida familiar: cohesión y adaptabilidad familiar. La cohesión es definida según dos componentes: el vínculo emocional y el nivel de autonomía ${ }^{25,29}$. La adaptabilidad es precisada como la habilidad del sistema familiar para cambiar sus estructuras de poder, relaciones de roles y reglas de relación en respuesta al estrés situacional y evolutivo ${ }^{30}$.

c) Cuestionario de salud general GHQ 12- Golbrerg: para estimar la salud mental de los padres, evalúa la percepción del individuo sobre su estado de bienestar general ${ }^{31}$, alcanzando altos niveles de confiabilidad $0,89^{32}$.

d) Ficha de selección y caracterización de la muestra: elaborada por los autores(as), en ésta se registran datos demográficos como edad, sexo, escolaridad, diagnóstico, fecha de ingreso, tipo de tratamiento recibido, antecedentes relevantes familiares, historia familiar de estresores familiares (como consumo de sustancias, abuso sexual, conflictos legales, muerte de un familiar, intento o suicidio de un familiar, violencia intrafamiliar, trastorno psiquiátrico de familiar).

Análisis de datos. Para este procedimiento se utilizó el Programa Estadístico SPSS 10.0. Se efectuaron cálculos de correlación, utilizando las pruebas de Punto Biserial y Chi-cuadrado, además del análisis de regresión lineal múltiple.

\section{Resultados}

Los resultados obtenidos en esta investigación indicaron por medio del inventario de depresión de Beck, que 10 adolescentes presentaron depresión leve, 15 , moderada y 7 , grave.

Al analizar la asociación de los factores de riesgo familiares con el intento suicida de adolescentes con trastorno depresivo, los análisis no arrojaron asociaciones estadísticamente significativas $\mathrm{p}<0,05$. Sin embargo, al efectuar los cálculos con la ideación suicida, se encontraron asociaciones por un lado con la adaptabilidad familiar adolescente $(r=0,44 ; p=0,01)$ y por otro, con el intento suicida de un familiar $(\mathrm{r}=0,36 ; \mathrm{p}=0,05)$ (Tablas 1, 2 y 3).

Como un intento de obtener un mayor análisis clínicamente relevante, se examinó la capacidad predictiva de las variables estudiadas. De esta forma, se investigó si alguna de las variables podía predecir, y en qué cantidad, las dos variables 
Tabla 1. Análisis de las asociaciones entre variables estresores familiares e ideación e intento suicida

\begin{tabular}{|c|c|c|c|}
\hline \multicolumn{2}{|c|}{$\begin{array}{l}\text { Intento suicida y variables estresores familiares } \\
\chi^{2}\end{array}$} & \multicolumn{2}{|c|}{$\begin{array}{l}\text { Ideación suicida y variables estresores familiares } \\
\mathbf{r}\end{array}$} \\
\hline Consumo de sustancias & $\begin{array}{r}0,005 \\
(0,94)\end{array}$ & Consumo de sustancias & $\begin{array}{r}-0,09 \\
(0,61)\end{array}$ \\
\hline Abuso sexual & $\begin{array}{r}0,008 \\
(0,92)\end{array}$ & Abuso sexual & $\begin{array}{r}0,08 \\
(0,65)\end{array}$ \\
\hline Conflictos legales & $\begin{array}{r}2,169 \\
(0,14)\end{array}$ & Conflictos legales & $\begin{array}{r}0,13 \\
(0,48)\end{array}$ \\
\hline Muerte de un familiar & $\begin{array}{r}0,744 \\
(0,38)\end{array}$ & Muerte de un familiar & $\begin{array}{r}-0,27 \\
(0,13)\end{array}$ \\
\hline Intento suicida de un familiar & $\begin{array}{r}0,112 \\
(0,73)\end{array}$ & Intento suicida de un familiar & $\begin{array}{r}0,36^{*} \\
(0,05)\end{array}$ \\
\hline Violencia psicológica & $\begin{array}{l}1,71 b \\
(0,19)\end{array}$ & Violencia psicológica & $\begin{array}{r}0,16 \\
(0,39)\end{array}$ \\
\hline Violencia física & $\begin{array}{r}0,536 \\
(0,46)\end{array}$ & Violencia física & $\begin{array}{r}0,17 \\
(0,35)\end{array}$ \\
\hline Trastorno psiquiátrico de un familiar & $\begin{array}{r}0,005 \\
(0,96)\end{array}$ & Trastorno psiquiátrico de un familiar & $\begin{array}{r}-0,01 \\
(0,88)\end{array}$ \\
\hline
\end{tabular}

*p $<0,05$.

Tabla 2. Correlaciones Punto Biserial con intento suicida y variables de adaptabilidad y cohesión familiar desde la perspectiva de padres y adolescentes

\begin{tabular}{|lccc|}
\hline Cohesión adolescentes & Cohesión padres & Adaptabilidad adolescentes & Adaptabilidad padres \\
\hline$-0,21$ & $-0,07$ & $-0,19$ & $-2,94$ \\
$(0,25)$ & $(0,63)$ & $(0,26)$ & $(0,99)$ \\
\hline
\end{tabular}

Tabla 3. Correlaciones Punto Biserial con ideación suicida y variables de adaptabilidad y cohesión familiar desde la perspectiva de padres y adolescentes

\begin{tabular}{|lccc|}
\hline Cohesión adolescentes & Cohesión padres & Adaptabilidad adolescentes & Adaptabilidad padres \\
\hline 0,18 & $-0,07$ & $0,44^{* *}$ & $-0,1$ \\
$(0,33)$ & $(0,17)$ & $(0,01)$ & $(0,55)$ \\
\hline
\end{tabular}

${ }^{* *} \mathrm{p}<0,01$.

dependientes que nos interesan; intento suicida e ideación suicida. Adicionalmente y dado que la evidencia muestra una relación entre ambas variables, se procedió a controlar el posible efecto de la ideación en el análisis del intento. De esta manera, el estudio estadístico mostró que la adaptabilidad familiar (adolescente) tiene un valor predictivo para la ideación suicida $\left(R^{2}=0,194 ; p=0,011\right)$ y para el intento suicida $\left(\mathrm{R}^{2}=0,29 ; \mathrm{p}=0,019\right)$, controlando el efecto de la ideación (Tabla 4). 
Tabla 4. Predictores intento suicida e ideación suicida en adolescentes

\begin{tabular}{|lcc|}
\hline \multicolumn{2}{|l|}{ Predictores de ideación suicida } & \\
\hline Variable & $\mathrm{R}^{2}$ & $\mathrm{p}$ \\
Adaptabilidad FACES adolescentes & 0,194 & $0,011^{* *}$ \\
Predictores de intento suicida con la variable ideación suicida como variable controlada & \\
\hline Variable & $\mathrm{R}^{2}$ & $\mathrm{p}$ \\
Adaptabilidad FACES adolescentes & 0,29 & $0,019^{*}$ \\
\hline
\end{tabular}

Variables predictoras: (Constante), cantidad de estresores, Adaptabilidad FACES Adolescentes, índice Goldberg, Cohesión FACES Padres, Adaptabilidad FACES Padres, Cohesión FACES Adolescentes.

*p $<0,05 . \quad$ *** $\mathrm{p}<0,01$.

Respecto al nivel de salud mental de los padres y su asociación con el intento suicida en adolescentes con trastorno depresivo, no se verificó relación estadística significativa (Tabla 5).

Referente a la cantidad de estresores familiares, recurriendo a la prueba de Punto Biserial, no se detectó una correlación estadísticamente significativa entre esta variable y el intento suicida $(r=0,07 ; p=0,67)$.

Además, se utilizó los coeficientes de correlación de Fischer y Phi, para analizar de forma independiente los estresores familiares con el intento suicida. Bajo estos análisis, no se encontraron asociaciones.

Por otro lado, al considerar la ideación suicida como variable dependiente, y cada estresor familiar de forma independiente, utilizando prueba Punto Biserial, se detectó una correlación positiva y moderada estadísticamente significativa con la variable intento suicida de un familiar $(r=0,35 ; \mathrm{p}$ $=0,045)$. En la mayoría de los casos era la madre del adolescente quien había tenido esta conducta.

Respecto al funcionamiento familiar (adaptabilidad y cohesión) y el intento suicida adolescente no se obtuvieron correlaciones (prueba Punto Biserial), tanto desde la perspectiva de los padres como de los adolescentes.

Al relacionar esta variable con ideación suicida, se encontró una correlación positiva y moderada, con significancia estadística (prueba Punto Biserial) para la variable de adaptabilidad familiar percibida por los adolescentes $(r=0,44 ; \mathrm{p}=0,01)$.

A modo de estimar de mejor manera los resultados y saber de qué forma se puede predecir el intento suicida se realizaron dos análisis de regresión. Al efectuar un primer análisis de regre-

Tabla 5. N ivel de salud mental de los padres y presencia 0 ausencia del intento suicida en los adolescentes

\begin{tabular}{|lccc|}
\hline $\begin{array}{l}\text { Tabla de contingencia Intento suicida * Indice Goldberg } \\
\text { Intento suicida }\end{array}$ & $\mathbf{- g}$ & $\mathbf{+ g}$ & Total \\
\hline NO & 7 & 10 & 17 \\
SI & 3 & 12 & 15 \\
Total & 10 & 22 & 32 \\
Chi $^{2}=1,66$ & & & \\
\hline
\end{tabular}

( $+\mathrm{g}=$ Deterioro en la salud mental de los padres)

(-g =Ausencia de deterioro en la salud mental de los padres) 


\section{Tabla 6. Relación entre la ideación suicida y las variables de adaptabilidad familiar desde la perspectiva de los adolescentes}

\begin{tabular}{|lcccc|}
\hline Predictores de ideación suicida & & & \\
\hline Variable & $\mathrm{R}^{2}$ & $\mathrm{~B}$ & $\mathrm{~T}$ & $\mathrm{P}$ \\
Adaptabilidad FACES adolescentes & 0,194 & 0,442 & 2,7 & $0,011^{* *}$ \\
\hline
\end{tabular}

Variables predictoras: (Constante), cantidad de estresores. Adaptabilidad FACES adolescentes, índice Goldberg. Cohesión FACES padres, adaptabilidad FACES padres. Cohesión FACES adolescentes **: $\mathrm{p}<0,01$.

sión lineal múltiple con las variables familiares en estudio y la ideación, se encontró una asociación estadísticamente significativa con la adaptabilidad familiar (adolescente), explicando 19\% de su variabilidad (Tabla 6).

Finalmente, en el segundo análisis de regresión lineal múltiple, se consideró la ideación suicida como co-variable. La adaptabilidad familiar (adolescente) resultó un predictor del intento suicida, explicando $29 \%$ de su variabilidad, con una adecuada significación estadística $\left(\mathrm{R}^{2}=0,29 ; \mathrm{p}=0,019\right)$.

Si bien no es posible afirmar que a mayor adaptabilidad familiar, existirá mayor probabilidad de llevar a cabo un intento suicida, se supone la presencia de una relación positiva entre ambas variables; puesto que, como se confirmó estadísticamente en este estudio, existe una relación positiva entre ideación suicida y adaptabilidad familiar (adolescente).

\section{DisCUSIÓN}

Los principales resultados obtenidos del estudio tienen relación con que la ideación suicida del adolescente correlaciona moderadamente con el intento suicida de un familiar y con la adaptabilidad familiar adolescente. Además, se encontró que controlando el efecto de la ideación suicida, la adaptabilidad familiar adolescente está asociada al intento suicida.

Lo anterior debe ser considerado cuidadosamente, puesto que si bien los resultados obtenidos son estadísticamente significativos, las correlaciones entre las variables son moderadas.

Lo encontrado resalta la importancia de dos tipos de variables relacionadas con el intento suicida, éstas son por una parte una variable estrechamente ligada a nivel cognitivo como es la ideación suicida y la otra referida a factores medioambientales y de contexto como es el funcionamiento familiar.

La asociación entre ideación suicida adolescente y el intento suicida de un familiar ha sido estudiada por otros autores, quienes señalan que en estas familias podría existir una forma de aprendizaje, proveyendo un modelo de solución ante dificultades emocionales o un modo de enfrentar la angustia ${ }^{33}$.

Por otra parte, un mayor nivel de adaptabilidad familiar (adolescente), resulta ser un factor de riesgo para la ideación suicida. Una mayor adaptabilidad significa, desde el modelo Circumplejo de Olson, Russell y Sprenkle (del cual se sustenta el presente estudio) que el sistema familiar opera de forma desestructurada para cambiar sus estructuras de poder, relaciones de roles y reglas, ausencia de control paterno y disciplina poco efectiva ${ }^{28}$ teniendo diversos efectos en el bienestar físico y emocional de sus integrantes. Además, en la etapa del ciclo vital familiar en la cual existe un miembro adolescente es necesario que el sistema reactualice sus roles y límites ${ }^{34}$, adaptándose a las nuevas fuentes de estrés que implica tener un miembro en esta etapa. Si la familia presenta dificultades en este proceso, tendiendo a una estructura caótica, puede traer consecuencias negativas para el adolescente ${ }^{35}$, como la observada en este estudio.

Además, un mayor nivel de adaptabilidad familiar (adolescente), independientemente del nivel de ideación suicida, resultaría ser un factor de riesgo para el intento, lo que se puede asociar con que la mayoría de los intentos suicidas 
adolescentes serían impulsivos ${ }^{36}$ apreciándose una baja intención de morir ${ }^{8}$.

De esta manera, el presente estudio contribuye a apreciar la interacción e influencia entre variables individuales (conductas y cogniciones de los adolescentes), con variables ambientales (el funcionamiento familiar y el intento suicida de un familiar), dando cuenta del carácter complejo y multicausal de este fenómeno. Los resultados encontrados en este estudio son coincidentes con los de otras investigaciones internacionales ${ }^{37,38}$

Estos hallazgos, si bien se tratan de correlaciones moderadas entre variables, entregan información relevante para analizar y en el futuro intentar establecer un modelo predictivo del intento suicida en adolescentes con trastorno depresivo. En cuanto a las limitaciones de este estudio, una de ellas se vincula al tamaño reducido de la muestra. Este aspecto debe ser considerado al menos en dos sentidos. En primer lugar se debe mencionar la dificultad encontrada para acceder a las personas que tuvieran las características requeridas. En segundo lugar, el alto nivel de rechazo de la muestra constituida por sujetos, que aun cumpliendo los criterios, no accedieron a participar. Esto se

\section{REFERENCIAS}

1. BRENT D. Antidepressants and Suicidal Behavior: Cause or Cure? Am J Psychiatry 2007; 164: 989-92.

2. King C, Kramer A, Preuss L, Kerr D, Weisse L, Venkataraman S. Youth-Nominated Support Team for Suicidal Adolescents: A randomized controlled trial. J Consult Clin Psychol 2006; 74: 199-206.

3. Pelkonen M, Marttunen M. Child and adolescent suicide. Pediatr Drugs 2003; 5: 243-65.

4. Almonte C. Conducta suicida en la niñez y adolescencia. En: Almonte C, Montt M, Correa A. Psicopatología infantil y de la adolescencia. Santiago, Chile: Editorial Mediterráneo Ltda.; 2003; 343-52.

5. Larraguibel M, González P, Martínez V, Valenzuela R. Factores de riesgo de la conducta suicida en niños y adolescentes. Rev Chil Pediatr 2000; 71: 183-91.

6. Instituto Nacional de la Juventud (INJUV). Suicidio juvenil: características y significados asociados. Santiago:Instituto Nacional de la Juventud; 1996.

7. Beautrais A, Mishara B. World Suicide Prevention: Suicide prevention across the life span. Crisis 2007; 28: 57-60

8. De la Barra F. Conductas suicidas en niños y adolescentes. Pediatr día 1989; 5: 152-6. debe considerar al momento de generalizar los resultados a una población mayor y comprender las conclusiones. Los hallazgos expuestos deben ser tomados como una primera aproximación y se sugiere replicar este estudio en futuras investigaciones con una muestra superior considerando las dificultades encontradas en el presente estudio.

Adicionalmente, la muestra correspondió a un solo nivel socioeconómico (bajo), siendo necesario ampliar a los distintos estratos, así como a otras variables sociodemográficas (edad, sexo, etc.). Igualmente en este estudio se controló el nivel de salud mental de los padres mediante un solo instrumento. Se sugiere ampliar a otros sistemas de medición para padres que incluyan mayor tipo de sintomatología.

Los resultados obtenidos también evidencian la importancia de integrar y contextualizar el fenómeno del suicidio, siendo necesario incluir a la familia en aspectos relacionados con la reducción y detección de los factores de riesgo asociados al intento suicida en adolescentes, y a su incorporación en el trabajo terapéutico de jóvenes con dicha conducta ${ }^{7,39}$.

9. Mitchell A, Kim Y, Pringerson H. Complicated grief in survivors of suicide. Crisis 2004; 25: 12-8.

10. Organización Mundial de la Salud (OMS). Prevención del suicidio un instrumento para docentes y demás personal institucional. Trastornos mentales y cerebrales. Departamento de Salud Mental y Toxicomanía. Ginebra: OMS; 2001. Disponible en http: //whqlibdoc.who.int/hq/2000/WHO_MNH_MBD_00.3_spa.pdf [consultado el 14 de Septiembre de 2007].

11. UlloA F. Prevención del suicidio en niños y adolescentes. Rev Chil Pediatr 1994; 3: 178-83.

12. World Health Organization (WHO). Preventing suicide: a resource for counsellors. Departament of mental healt and substance abuso. Management of mental and brain disordens World Healt Organization. Geneva: WHO; 2006. Disponible en: http: // www.who.int/es/http:whqlibdoc.who.int/publications/2006/9241594314spa.pdf [consultado el 14 de septiembre de 2007].

13. Ministerio de SAlud (MinSAL). Programa nacional de diagnóstico y tratamiento de la depresión severa. 2004. Disponible en: http://www.minsal.cl/ http://www.redsalud.gov.col/temas_salud/proteccion/saludmental3.html [consultado el 14 de Septiembre de 2007]. 
14. REYes W, TORRes N. Intento suicida y funcionamiento familiar. Rev Cubana Med Gen Integr 2001; 17: 452-60.

15. Lyon M, Benoit M, O'Donnell R, Getson P, Silber T, Walsh T. Assessing African American Adolescents' Risk for Suicide Attempts: Attachment Theory. Adolescence 2000; 35: 121-34.

16. Diamond G, Didner H, Waniel A, Priel B, Asherov J, Arbel S. Perceived parental care and control among israeli female adolescents presenting to emergency rooms after self-poisoning . Adolescence 2005; 40: 257-63.

17. Madu S, Matla M. Family environmental factors as correlates for adolescent suicidal bahaviors in the limpopo province of South Africa. SBP-Journal 2004; 32: 341-54.

18. BRENT D. Risk factors for adolescent suicide and suicidal behavior: Mental and substance abuse disorders, family environmental factors, and life stress. Suicide Life Threat Behav 1995; 25: 52-63.

19. American Academy of Child and Adolescent Psychiatry (AACHAP). Suicide and suicide attempts in adolescents. Pediatrics 2000; 105: 1-4.

20. Portes P, Sanghu D, Longwell-Grice R. Understanding adolescent suicide: a psychosocial interpretation of developmental and contextual factors. Adolescence 2002; 3: 805-15.

21. Mann J, Bortinger J, Oquendo M, Currier D. Historia familiar del comportamiento suicida y los trastornos emocionales. Am J Psychiatry 2005; 162: 1672-9.

22. Silva H, Martínez J. ¿Es efectivo que los antidepresivos aumentan el riesgo de suicidio? Rev Med Chile 2007; 135: 1195-201.

23. Beck A, Rush A, Shaw B, Emery G. Terapia cognitiva de la depresión. Bilbao: Descleé de Brouwer; 1983.

24. Sanz J, Vásquez C. Fiabilidad, validez y datos normativos del Inventario para la depresión de Beck. Psicothema 1998; 10: 303-18.

25. Polaino-Lorente A, Matínez P. Evaluación psicológica y psicopatológica de la familia. Madrid: Ediciones RIALP. 1998.

26. Zegers B, Larrain M, Polaino-Lorente A, Trapp A, Diez I. Validez y confiabilidad de la versión española de la Escala de Cohesión y Adaptabilidad Familiar (CAF) de Olson, Russell \& Sprenkle para el diagnóstico del Funcionamiento Familiar en la población chilena. Rev Chil Neuro-Psiquiat 2003; 41: 39-54.
27. Olson D, Russell C, Sprenkle D. Circumplex model of marital and family system I: cohesión and adaptability dimensions, family types and clinical applications. Fam Process 1979; 18: 3-28.

28. Olson DH, Portner J, Bell RQ. FACES II: Family adaptability and cohesion evaluation scales. Saint Paul: Family Social Science. University of Minnesota. 1982.

29. Cusinato M. Psicología de las relaciones familiares. Barcelona: Herder; 1992.

30. López S. El FACES II en la evaluación de la Cohesión y Adaptabilidad Familiar. Psicothema 2002; 14: 15966.

31. García C. Manual para la utilización del Cuestionario de Salud General de Goldberg, adaptación cubana. Rev Cubana Med Gen Integr 1999; 15: 88-97.

32. Araya R, Wynn R, Lewis G. A comparison of two psychiatric case finding questionnaires (GHQ-20 and SRQ-20) in primary care in Chile. Soc Psychiatry Psychiatr Epidemiol 1992; 27: 168-73.

33. Rubistein J, Halton A, Kasten L, Rubin C, stechler G. Suicidal behavior in adolescent: stress and protection in different family contexts. Am J Orthopsychiatry 1998; 68: 274-84.

34. Florenzano R. Familia y salud de los jóvenes. Santiago: Ediciones Universidad Católica de Chile; 1995.

35. Irribarne M. Desarrollo psicológico del adolescente. En: Almonte C, Montt M, Correa A Psicopatología infantil y de la adolescencia. Santiago, Chile: Editorial Mediterráneo Ltda.; 2003; 27-33.

36. Bloch D. Adolescent suicide as a Public Health Threat. J Child Adolesc Psychiatr Nurs 1999; 12: 2638.

37. Lee M, Wong B, Chow B, McBride-Chang C. Predictors of suicide ideation and depression in Hong Kong adolescents: perceptions of academic and family climates. Suicide Life Threat Behav 2006; 36 : 82-97.

38. Kerr D, Preuss L, King CH. Suicidal adolescents, social support from family and peers: genderspecific associations with Psychopathology. J Abnorm Child Psychol 2006; 34: 103-14.

39 Orbach I. From abandonment to simbiosis: a developmental reversal in suicidal adolescents. Psychoanal Psychol 2007; 24: 150-66. 